\title{
La gestión de la innovación y de la transferencia de tecnologías. Experiencia de la estación experimental "Indio Hatuey"
}

\author{
The management of innovation and technology transfer. Experience of the \\ Experimental Station "Indio Hatuey" \\ Lamela L, Simón L, Suárez J y Pérez $\mathrm{A}^{1}$ \\ ${ }^{1}$ Estación Experimental de Pastos y Forrajes "Indio Hatuey" \\ Central España Republicana CP 44280, Matanzas, Cuba \\ luis.lamela@indio.atenas.inf.cu
}

CONFERENCIA DEL I SEMINARIO INTERNACIONAL DE GANADERÍA AGROECOLÓGICA REALIZADO EN VILLAVICENCIO COLOMBIA, 11 Y 12 DE NOVIEMBRE DE 2010

\section{RESUMEN}

La ganadería cubana, al igual que la del resto de Latinoamérica, necesita tecnologías novedosas que sean sostenibles desde los puntos de vista ambiental, socioeconómico y técnico-productivo, si se considera la definición de Desarrollo Sostenible brindada por la FAO (1990). Algunas de estas tecnologías son los Sistemas Silvopastoriles y la relativa a la producción, beneficio y conservación de semillas de plantas forrajeras, desarrolladas por la Estación Experimental de Pastos y Forrajes "Indio Hatuey" para la ganadería. Asimismo, Cuba es un país con escasos recursos energéticos y se ve obligada a importar casi todo el petróleo que necesita para mantener su producción. Por ello, una vía para el país lo constituye el desarrollo de sistemas sustentables de producción pecuaria como los mencionados anteriormente, que dependan de los recursos locales que estén disponibles. El país destina más de 2’500.000 hectáreas de pastizales para la ganadería, pero su baja productividad constituye un problema que es necesario solucionar para producir suficiente cantidad de leche y carne destinada a una población que ha ido en constante aumento. Los pastos mejorados, en la década de los años 80 , llegaron a superar el 50\% del área agrícola de la ganadería en el país, pero actualmente solo alcanzan el 19\%. El $58 \%$ está cubierto por pastos naturales y por malezas, entre las que se destacan 
el aroma y el marabú, que cubren el $39 \%$ del área agrícola total de los pastizales. No menos importante es también el alto porcentaje que hoy ocupan el espartillo y el caguazo. En la actualidad la fertilidad de los suelos ganaderos decrece, y como consecuencia, la producción de biomasa de los pastizales ha disminuido y los pastos se han degradado. Esto ha traído serias consecuencias para la ganadería vacuna, al reducirse los rendimientos de leche y carne e incrementarse los costos de producción animal. Las causas de este deterioro son múltiples, entre las que se destacan el pastoreo excesivo, la disminución de la fertilidad del suelo, el pobre papel que han desempeñado las leguminosas herbáceas en los pastizales, la invasión de malezas, las sequías y la erosión. La renovación y la recuperación de los pastizales deben constituir la tarea fundamental para disponer, a corto y mediano plazo, de una base alimentaria en cantidad y calidad suficientes para sostener la masa bovina, y por ende, contribuir eficazmente a la recuperación de la ganadería.

Palabras clave: Biodiversidad, tecnología, innovación.

\section{ABSTRACT}

Cuban livestock, like the rest of Latin America needs innovative technologies that are sustainable in environmental, economic and technical productive, considering the definition of sustainable development provided by FAO (1990). Some of these technologies are silvopastoral systems and on the production, processing and conservation of fodder plant seed, developed by the Experimental Station of Pastures and Forages "Indio Hatuey" for livestock. Likewise, Cuba is a country with scarce energy resources and is forced to import nearly all the oil it needs to maintain its production. Therefore, a way for the country is the development of sustainable livestock production systems such as those mentioned above, which depend on local resources available. The country spends more than 2500000 hectares of pasture for livestock, but its low productivity is a problem that needs solving to produce enough milk and meat for a population that has been steadily 
increasing. Improved pastures in the mid-80's, reaching over $50 \%$ of the agricultural area of livestock in the country, but currently only reach $19 \% .58 \%$ is covered with natural grasses and by weeds, among which stand the smell and marabou, covering $39 \%$ of the total agricultural area of grassland. No less important is also the high percentage who now occupy the espartillo and caguazo. At present, the soil fertility decreases and livestock as a result, biomass production of grasslands has declined and pastures have been degraded. This has serious consequences for the cattle industry, by reducing meat and milk yields and increased costs of animal production. The causes of this decline are many, among which include overgrazing, declining soil fertility, poor role played by herbaceous legumes in pastures, weed invasion, drought and erosion. The renewal and recovery of pastures should be the fundamental task to provide short to medium term, of a food base in sufficient quantity and quality to sustain the mass cattle and thus contribute effectively to the recovery livestock.

Keywords: Biodiversity, technology, innovation.

\section{INTRODUCCIÓN}

La ganadería se desarrolla en un contexto global donde cada vez gana más importancia la gestión de la innovación, la cual no tiene por qué estar ajena a esta actividad y puede desempeñar un importante papel en la adecuación de las tecnologías en el medio ambiente del productor, evitando volver a caer en el peligro de la "modernización" de la agricultura que estuvo asociada a la Revolución Verde de los años 60. Por ello, hablar de la introducción de la gestión de la innovación en la agricultura, y específicamente en la ganadería, coincide con la nueva visión, en la cual la interacción hombre-naturaleza no es una simple gestión económica, sino que integra los procesos geológicos, fisicoquímicos, biológicos, económicos y sociales; por lo tanto, los sistemas de producción que se desarrollen deben ser sostenibles desde el punto de vista productivo-económico-ecológico y, además, socialmente justos. 
La necesidad de una agricultura sostenible en el Tercer Mundo, e incluso en todo el planeta, está potenciada por el hecho de que el problema de los países en vías de desarrollo no es la transferencia de tecnologías, agravada por la inexistencia de recursos financieros, sino la transferencia de tecnologías adecuadas ambientalmente, pues las tierras y la mayoría de las tecnologías actualmente en uso no son apropiadas para afrontar los requerimientos presentes y mucho menos los futuros. Durante varias décadas se ha promovido un estilo de agricultura que oferta paquetes tecnológicos de un alto costo y asociados a insumos deficitarios para la agricultura de los países no desarrollados, por lo que una agricultura de este tipo no es una alternativa real para el Tercer Mundo, según lo planteado por la FAO, (1993).

Pero no sólo debe haber transferencia de tecnologías sostenibles desde el Norte hacia el Sur, sino también en el otro sentido, hacia los países desarrollados, pues existen tecnologías en armonía con la naturaleza. Este cambio de polaridad de la transferencia requiere nuevos procedimientos para lograr un flujo de tecnologías e información, por lo que es necesario comenzar por la transferencia tecnológica adecuada dentro de la propia agricultura nacional, en este caso la cubana, la cual estaba acostumbrada a un suministro permanente de tecnologías e insumos foráneos. Ello implicó que la EEPF "Indio Hatuey" considerara desarrollar un nuevo enfoque para la generación, transferencia y mejoramiento de tecnologías apropiadas para el productor primario, lo cual exigía tener en consideración sus necesidades y utilizar métodos asociados a la innovación.

\section{¿QUÉ SIGNIFICA INNOVACIÓN?}

En la literatura existen diversas definiciones, pero se escogió la brindada por Pavón y Goodman, (1976) quienes consideran que la innovación es "el conjunto de actividades, inscriptas en un determinado período de tiempo y lugar, que conducen a la introducción con éxito en el mercado, por primera vez, de una 
idea en forma de nuevos o mejores productos, servicios o técnicas de gestión y organización", en coincidencia con Benavides, (1998) quien precisa que esta variedad de ofertas al mercado (los clientes) también incluye los productos intangibles. Esta vinculación innovación-mercado es también defendida por autores como Gee, (1981) y Piatier, (1987); ello se debe a que es necesario apreciarla en el marco de una concepción integrada del ciclo de vida del producto, en la cual se produce una dinámica coherencia entre el campo tecnológico y el campo de las organizaciones productivas, que se manifiesta, según Ait-El-Hadj, (1990), a través de la concepción del producto: nivel de servicios prestados al cliente en la realización de una función (Figura 1).

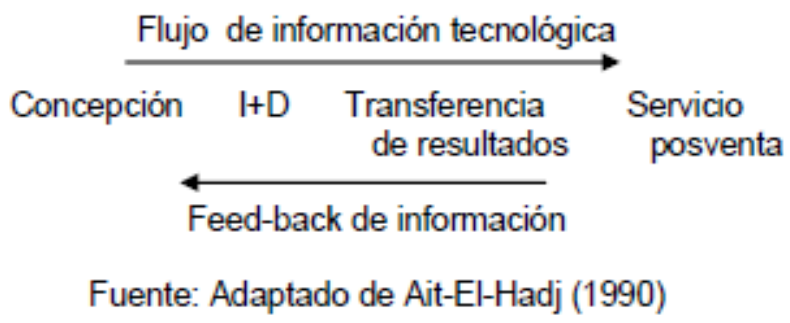

Figura 1. Concepción integrada del ciclo de vida del producto.

Todo ello hace que resulte evidente la existencia de un punto de encuentro entre los cedentes (los centros de investigación) y los receptores (las empresas ganaderas) de tecnologías, es decir, la presencia de un mercado de tecnologías, al cual concurren ambos tipos de organizaciones y donde la demanda es puntual. Pero esa tecnología que se oferta ha de especificarse desde el punto de vista del cliente, o sea, adecuarse a sus realidades para que realmente tenga utilidad, criterio sostenido por Drucker, (1986).

Lo anterior no es más que adoptar un enfoque de mercado (un market pull) envés de un technology push. Dicho enfoque no sólo tiene que aportar la tecnología que necesita el agricultor, sino que ésta tiene que estar acompañada de otros beneficios, o sea, de un valor agregado, como es el caso de un buen servicio posventa de consultorías relativo al perfeccionamiento de la tecnología, 
la preparación del personal que la explotará, la entrega de la documentación necesaria, etc. Ello exige que la organización científica que genera la tecnología posea una cultura organizativa que le permita examinar continuamente en qué forma puede ofrecer mayores ventajas a sus clientes en armonía con el medio natural; realizar una coordinación interfuncional, en el seno de la organización, de sus recursos y capacidades en la creación de beneficios para sus clientes; así como mantener con estos una relación a largo plazo que intensifique los vínculos en el tiempo. Ello quiere decir que tiene que existir una vinculación estrecha entre el marketing y la innovación, que son las dos funciones esenciales para lograr el papel de una empresa: crear clientes, como planteara Drucker, (1954). Esto es reforzado por estudios realizados en empresas norteamericanas, los cuales han señalado que los dos subsistemas organizativos más importantes son el marketing y la I+D (Bueno y Pablo, citados por Morcillo, 1989).

Es evidente que dichas innovaciones no pueden ser sólo de productos o de procesos, sino también deben incluir innovaciones comerciales y de gestión. La innovación comercial puede provenir de la modificación de las distintas variables del marketing (producto, precio, promoción y canales de distribución), ya que el marketing que practica la empresa puede ser la forma más decisiva de diferenciación, como considera Levitt, (1980); mientras que la innovación de gestión está asociada, en opinión de Benavides, (1998) con la adopción de una dirección estratégica que anticipe, evalúe y determine la dirección e intensidad de los cambios ocurridos en el sistema y, si es posible, que contribuya con los procesos de innovación al propio cambio, fruto del análisis de sus puntos fuertes y débiles y de las necesidades del mercado. Según Benavides, (1998) la innovación surge a partir de la decisión estratégica de lanzar al mercado un resultado de la investigación y constituye el primer paso de la difusión (transferencia) de un nuevo producto o servicio, para lo cual es necesaria la 
creación de nuevos canales de distribución o la adecuación de los existentes, así como desarrollar acciones de promoción.

En el caso de los sistemas silvopastoriles, la tecnología que se utilizó como ejemplo inicial y que, incluso, implica un cambio de forma de pensar y actuar en la ganadería cubana, se hizo necesario un conjunto de innovaciones comerciales y de gestión (organizativas) durante el proceso de transferencia, sin las cuales los resultados hubiesen sido diferentes. Estas innovaciones, que llevaron al éxito de la tecnología, han sido:

- Invitar a la estación a directivos y productores directos, con el objetivo de que recibieran un adiestramiento teórico-práctico sobre el establecimiento y la explotación de la tecnología en condiciones de campo.

- La realización de recorridos periódicos en las unidades productivas que establecen o explotan la tecnología, por parte de experimentados investigadores. Su objetivo es interactuar con los productores y brindarle seguimiento al proceso de adopción.

- La preparación de los profesores de la Escuela de Capacitación de la Agricultura en la provincia de La Habana para convertirlos en difusores tecnológicos.

- La retirada del rebaño de las unidades de producción seleccionadas, con el objetivo de que sus trabajadores se dedicaran completamente al establecimiento del sistema.

- La tecnología no rechazó, sino coexistió, con prácticas ganaderas arraigadas tales como: el uso de cercas vivas; el mantenimiento del estrato herbáceo existente que tuviese calidad, como es el caso del Panicum y el Cynodon; así como la rotación del rebaño, entre otras.

- La realización en varias ocasiones, y en diferentes regiones del país, de un Diplomado en Silvopastoreo destinado al personal profesional de las empresas ganaderas y a los investigadores, extensionistas y profesores. 
- El mejoramiento continuo de la tecnología a partir de la interacción entre investigadores y productores, e incluso el desarrollo de soluciones para cada unidad productiva.

Dichas innovaciones han estado potenciadas por un conjunto de factores que han sido decisivos para el éxito de la tecnología, sobre todo en la provincia de La Habana, pionera en su difusión y uno de los cuatro territorios que deciden la producción ganadera cubana. Los factores complementarios fueron:

- La superioridad de la tecnología con relación a las existentes, ya que brinda fiabilidad, calidad técnica, flexibilidad, así como facilidad de uso y de mantenimiento al productor, lo cual posibilita que éste tenga una credibilidad y confianza en el producto. Todo ello hace que dicha tecnología sea muy adecuada para las condiciones actuales de la ganadería cubana. Este tipo de superioridad se demuestra en un estudio realizado en 1990 por Holling y Pugh (citados por Arbonies, 1993), el cual sintetiza diferentes investigaciones sobre los factores que tienen mayor peso en las decisiones de compra 0 adopción de tecnologías. Este estudio aportó el siguiente orden: fiabilidad (con un gran peso), calidad técnica, mantenimiento, plazo de entrega, facilidad de uso, precio de venta y avance técnico.

- La prioridad dada a esta tecnología por parte de la dirección de la estación.

- La tecnología es de fácil comprensión y asimilación por parte de los clientes, lo cual se potenció con la capacitación y la interacción periódica investigadorproductor; tampoco provoca una alteración total del sistema productivo, en coincidencia con los criterios brindados por Mansfield, (1968) para el logro de la difusión de una innovación.

- El mantenimiento de relaciones a largo plazo con las empresas ganaderas, las delegaciones de la agricultura y el Vice-ministerio de Ganadería; la potenciación continua de esta cadena de relaciones efectivas y la imagen de la estación favorecieron la transferencia, ya que los activos asociados a 
determinada marca (en este caso "Indio Hatuey"), como son el prestigio, la reputación, el reconocimiento, la credibilidad y la confianza, son intangibles que aportan valor para los clientes.

- No se les creó a los productores falsas expectativas asociadas a fuertes mejoras radicales ni reducciones de los costos en un plazo reducido; por el contrario, se les planteó que los resultados serían graduales.

- El apoyo brindado por el MINAGRI en lo referente a la asignación de los insumos necesarios para la inversión (alambres, postes, combustible) en el establecimiento del nuevo sistema de producción.

- La reducción del plazo de establecimiento logrado a partir de la participación decisiva del propio productor y la asistencia periódica de los investigadores.

- La disminución del costo para el cliente, que radica en una reducción de los costos operacionales, de servicio y de mantenimiento, puesto que el animal asume parte de éste a partir de un adecuado manejo del rebaño y del pastizal.

- El hecho de involucrar a los productores como fuente de ideas innovadoras, por lo que estos desempeñaron no sólo un papel de clientes, sino otro más activo: el de socio-colaboradores.

- La razón de no dar por terminada la transferencia hasta que el sistema de producción esté a pleno rendimiento y lleve algún tiempo funcionando.

Todo este proceso de transferencia y mejoramiento de la tecnología ha traído consigo excelentes resultados productivos, reproductivos y económicos en las empresas donde se explota.

Por todo lo antes expuesto, en la EEPF "Indio Hatuey" se ha demostrado que una vía de generar, transferir y mejorar tecnologías agropecuarias sostenibles es aplicar un enfoque innovador que considere el papel activo del productor primario. Para ello se hizo necesario cambiar la visión existente, basada en un 
modelo centrado en la difusión por oferta de conocimientos, donde el sector científico decidía qué investigar y el cliente no participaba en la investigación y la transferencia (ambas eran monodisciplinarias, basadas en disciplinas y cultivos), para ir evolucionando hacia un modelo centrado en las demandas de los clientes, en el cual la investigación se decide en conjunto con éste, quién participa en la ejecución de los procesos de generación y transferencia, ambos multidisciplinarios (por productos).

Todo este cambio implica poner una mayor atención en la empresa, o sea, el lugar donde se aplica la tecnología y se logran los resultados productivos, criterio básico de la gestión de la tecnología y la innovación.

Para ello, Suárez y Pérez, (1999) sugirieron un modelo que caracteriza y se adecua al método empleado en el desarrollo y la transferencia de tecnologías en la EEPF "Indio Hatuey" (Figura 2), en el cual se muestra que debe existir un emisor de la tecnología, un mecanismo de transferencia y un receptor, entre los que debe haber una estrecha interrelación.

\section{CASO 1. TECNOLOGÍA DEL SILVOPASTOREO ESTRATEGIA SEGUIDA}

Las graves afectaciones que han tenido los recursos naturales y la actual crisis leconómica, han rehabilitado el interés por lograr un desarrollo acelerado y sostenido de la ganadería, el cual solo se alcanzará en la medida que las estrategias de producción sean congruentes con el uso racional del ecosistema.

Por otra parte, según Suárez y Simón, (1996) el proceso de innovación tecnológica tiene que adecuarse a las condiciones socio-culturales, biofísicas y económicas del productor, tener carácter multidisciplinario y un enfoque holístico del proceso de producción, así como darles participación a los productores y aprender de ellos. Por ello se hizo necesario, en el marco de la transferencia tecnológica, estudiar la influencia del silvopastoreo en el hombre y de éste sobre 
el sistema, lo que implicaba la evaluación del impacto económico, productivo, ambiental y organizacional, así como la capacitación y el grado de asimilación de los productores.

Las investigaciones desarrolladas en la EEPF "Indio Hatuey" demostraron, desde los 80, las potencialidades que poseen muchas leguminosas arbóreas por su alto contenido de proteína, así como las posibilidades de producir leche y carne bovina con el uso del silvopastoreo. Sin embargo, la introducción de estos resultados no fue posible mientras en el país existió una ganadería sustentada en la importación de alimentos, combustibles, pesticidas y fertilizantes, que al desaparecer provocaron un notable descenso de la producción ganadera.

Hoy existen las condiciones propicias para entender que el desarrollo de la ganadería se logrará mediante nuestros propios recursos y tecnologías, pues se ha demostrado que es posible incrementar la producción animal con la utilización de los pastos y los forrajes de leguminosas arbóreas, los primeros como base energética de la dieta y los segundos como alimentos de alta calidad que permiten una ración balanceada en energía, proteínas y minerales, ambos producidos en la propia unidad o finca y con efectos económicos ambientales y sociales apreciables.

\section{PROCEDIMIENTO SEGUIDO PARA LA TRANSFERENCIA DEL SILVOPASTOREO}

Para la transferencia de la tecnología del silvopastoreo se consideró una estrategia que motivara a los productores, mediante la demostración práctica de los resultados obtenidos en las investigaciones y validados en la producción, seguido de un proceso de capacitación y divulgación de los diferentes pasos del proceso tecnológico (Anon, 2009). En cuanto a la correcta aplicación de la tecnología, se creó un grupo para la asesoría y monitoreo de la actividad, con el 
fin de lograr además la retroalimentación entre el centro de investigación generador de la tecnología y los productores.

DIRECTIVOS INVESTIGADORES

Emisor

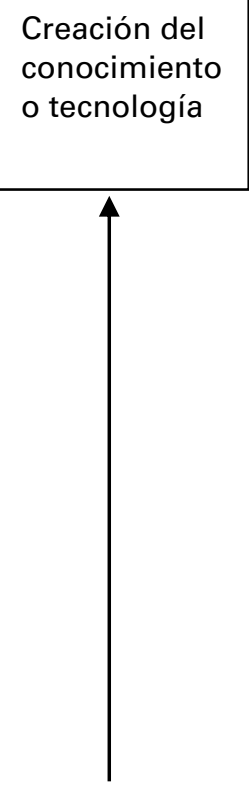

\section{Canal de} transmisión de información

PERSONAL TECNICO

OBREROS

Receptor

Proceso de comunicación

Adopción

y mejoramiento

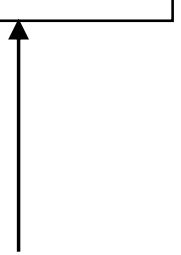

Innovaciones de producto, proceso y organizativas organizativas y de comercialización

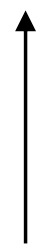

Formación del personal Científico productivo

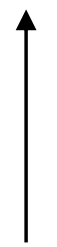

Formación del personal productivo y científico
Gestión Tecnológica

y de la Innovación

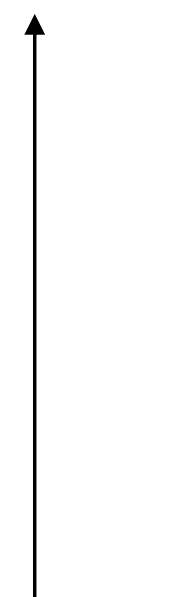

Formación

Figura 2. Modelo para la transferencia de las tecnologías

La introducción de estos sistemas de producción comenzó con la selección de las fincas para definir las variedades de pasto, las especies arbóreas, la transformación parcial o completa del área y la recuperación del cercado y las instalaciones de las unidades. Posteriormente, se realizó un laboreo mínimo parcial o total de la unidad y se efectuó la siembra de las arbóreas (generalmente Leucaena leucocephala) en franjas separadas (5 $\mathrm{m}$ de entresurco o calle) y a 1-2 m de narigón o a chorrillo ligero en surcos de muy poca 
profundidad, según la topografía del terreno, perpendicular a los rayos del sol o en curvas de nivel en las pendientes; de no existir el pasto mejorado (Panicum maximum, Cynodon nlemfuensis u otras gramíneas), se sembró en las calles.

\section{SIEMBRA Y LABORES CULTURALES}

Las labores de siembra y cultivo se realizaron de forma manual y con el auxilio de los bueyes por los propios obreros de las unidades, lo cual fue posible porque los animales fueron trasladados a otras fincas, donde la carga lo permitía. De esta forma, se garantizó que la fuerza de trabajo existente pudiera dedicarse totalmente a las labores de siembra y cultivo y al cercado, y con ello se logró acelerar el establecimiento de las leguminosas arbóreas en menos de un año, en dependencia de las condiciones edafo-climáticas y la época de siembra. Por su parte, la preparación de tierra se realizó con un mínimo de labores, que incluyeron la roturación y dos o tres pases de grada a las franjas según el terreno.

En lugares donde el pasto base estaba constituido por gramíneas mejoradas o cultivadas, como el pasto estrella, la guinea, la pangola o la bermuda, se utilizó la preparación de tierra en franjas de 2 ó $3 \mathrm{~m}$, lo que redujo el número de labores de aradura en un 40\% aproximadamente; el costo de la preparación varió entre 27 y 99 pesos/ha, en la cual se pudieron combinar perfectamente el uso del tractor y la tracción animal.

La preparación convencional de tierra se aplicó en lugares donde, además de las leguminosas arbóreas, era necesario sembrar gramíneas cultivadas y leguminosas herbáceas.

\section{REHABILITACIÓNDE PASTIZALES Y BIODIVERSIDAD}

La rehabilitación de los pastizales degradados de pasto estrella o guinea, mediante los métodos agrotécnicos empleados y el prolongado reposo de las 
áreas, sin la presencia de los animales, permitió la sucesión espontánea de estas gramíneas. Las leguminosas herbáceas autóctonas, que aportan alimento para el ganado y contribuyen a la fertilidad de los suelos, también resultaron favorecidas; los bancos de proteína de leucaena que estaban afectados por el manejo, se recuperaron sensiblemente. Especial atención recibieron las siembras y la reparación de las cercas vivas de Gliricidia, Erythrina y otros árboles, para potenciar la producción de biomasa. Además del Silvopastoreo, las vaquerías se completan con áreas complementarias de caña y de king grass para la suplementación de los animales en la época de seca. De esta biodiversidad vegetal el ganado puede obtener una ración balanceada en el potrero, además de poder contar, al cabo del tiempo, con la sombra necesaria.

\section{COSTO DEL ESTABLECIMIENTO Y RECUPERACIÓN DE LA INVERSIÓN}

Los costos desde la retirada de los animales (que debe coincidir con el inicio de la preparación de tierra) hasta el momento de comenzar la explotación nuevamente, aparecen en la Tabla 1. En la relación de gastos el componente salarial resultó el más elevado (67,6 y 44,3\% para cada alternativa), donde está implícito el valor de los postes y la labor de cercado. La amortización de la inversión se recuperó en el primer año de explotación a partir de la realización de la producción de leche y la obtención de los terneros.

Por otra parte, también fue posible recuperar la inversión durante el establecimiento de la leucaena, mediante la cosecha de la hierba de los entresurcos para heno y forraje o la siembra simultánea de cultivos agrícolas de ciclo corto de producción, como frijoles, calabaza, yuca, boniato y maíz, sembrados en surcos en las franjas. El intercalamiento de estos cultivos estimuló la participación de los vaqueros en las labores de limpieza, lo cual contribuyó al establecimiento de la siembra y a acelerar la amortización de la inversión. 
Tabla 1. Costo del establecimiento de ambas alternativas del silvopastoreo (\$/ha)

\begin{tabular}{lcc}
\hline \multicolumn{1}{c}{ Producto / labor } & Por franjas & Convencional \\
\hline Siembra de Leucaena & 7,98 & 7,98 \\
Inóculo & 0,83 & 0,83 \\
Siembra de guinea & - & 48,00 \\
Preparación de tierra & 27,31 & 99,25 \\
Salario & 153,52 & 153,52 \\
Alambre de púas (cercado) & 37,27 & 37,27 \\
\multicolumn{1}{c}{ Total } & 226,91 & 346,85 \\
\hline
\end{tabular}

IMPACTO EN EL ECOSISTEMA

Además de la rehabilitación de los pastizales y la sombra necesaria para el ganado, tan importante en las condiciones de clima tropical sin la necesidad de tener que utilizar instalaciones para este fin, la reducción de la radiación solar, la incorporación de nitrógeno y la retención de la humedad en el silvopastoreo influyen marcadamente en el fisiologismo de las gramíneas, ya que retardan considerablemente su maduración, lo que les permite ser manejadas en el potrero, conjuntamente con las leguminosas, sin afectar su calidad. Ejemplo de ello fueron los elevados valores de proteína encontrados en las gramíneas (entre 8 y $11 \%)$.

Por otra parte, las leguminosas volubles como Neonotonia, Centrosema, Teramnus e Indigofera encuentran en las arbóreas sus soportes o tutores vivos que les proporcionan las condiciones ideales para su desarrollo; mientras que las leguminosas arbóreas caducifolias como Albizia y Gliricidia actúan como incorporadoras de abono verde, con la caída de sus hojas al comienzo del invierno.

La utilización del franjeo disminuye el área de tierra que se debe mover, con lo que se evita la acción perjudicial de la aradura, aspecto muy importante si se tiene en cuenta que alrededor del $30 \%$ de los suelos del país están expuestos a 
la erosión, y de ellos, la mayor parte se dedica a la ganadería. Los árboles desempeñan un papel importante en la disminución de la erosión de los suelos, al evitar el impacto de la lluvia, y con sus raíces, los arrastres de las corrientes.

En cuanto a la fertilidad de los suelos, lo más significativo resultaron los contenidos de nitrógeno fácilmente hidrolizable (NFH) detectados en los sistemas arborizados, a los 3 años de sembrados, lo que evidencia un incremento en el reciclaje de este elemento. Además, se encontraron los mayores porcentajes de materia orgánica, calcio, magnesio, fósforo y potasio, particularmente en los sistemas silvopastoriles enclavados en los suelos Pardos tropicales.

La presencia de la macrofauna edáfica resultó significativamente superior en los sistemas arborizados; se destacó la presencia de las lombrices de tierra (clase Oligochaeta, orden Haplotaxida) y un notable crecimiento de los órdenes Coleoptera y Stylommathophora, como aporte a las características biológicas del suelo y de la fertilidad.

Por otra parte, los árboles son los organismos vivos que más eficientemente utilizan la energía solar a través de la fotosíntesis, y Cuba, como país tropical, cuenta con abundante sol y un rico germoplasma arbóreo forrajero. Los árboles, además, convierten el $\mathrm{CO}_{2}$ en materia orgánica, que en el caso del silvopastoreo, según los cálculos, se estima en 121,3 ton/ha/año como mínimo, con lo cual contribuyen a la descontaminación del aire y la reducción del efecto invernadero. Por otro lado, el hecho de rotar los animales durante un prolongado lapso de tiempo permite un aislamiento, y de esta forma, romper el ciclo de numerosos parásitos internos y liberar las áreas de agentes infecciosos causantes de enfermedades durante la etapa de establecimiento del silvopastoreo. 


\section{RESULTADOS}

La garantía de la sostenibilidad del silvopastoreo radica en el manejo de su explotación. El sistema debe comenzar su explotación con pocos animales (1,21,5 UGM/ha) y a medida que se desarrollan las arbóreas podrá ir asimilando una mayor carga, en dependencia de la composición del pastizal y la calidad de los suelos.

El descanso o reposo que requieren las arbóreas es de vital importancia (alrededor de 30-35 días en la época de lluvia y más de 65 días en seca), mientras que la ocupación de los cuartones no debe exceder de 4 días. Asimismo, el acuartonamiento se debe realizar a partir de estas indicaciones en el caso de las vacas lecheras, y con el ganado en desarrollo el número de cuartones que se utilizarán puede ser menor.

La leucaena se consideró establecida, y lista para comenzar a explotar, cuando sobrepasó la altura promedio de $2 \mathrm{~m}$; se iniciaron las mediciones de los distintos indicadores de la producción de leche durante 2 años consecutivos, con una muestra de 1.300 vacas lecheras, en su mayoría de la raza Siboney $(5 / 8$ Holstein X $3 / 8$ Cebú).

En cuanto a la leche, se analizó la producción a los 305 días de lactancia, así como la producción y duración por lactancia, cuyos resultados aparecen en la Tabla 2. Como se puede observar, la producción de leche a los 305 días de lactancia y en el total y duración de la lactancia resultó altamente significativa $(P<0,001)$ a favor del silvopastoreo cuando se comparó con el monocultivo de gramíneas (C. nlemfuensis y $P$. maximum), con lo cual se demostró la importancia de lograr la relación adecuada pasto-arbórea en el silvopastoreo para poder alcanzar una producción lechera satisfactoria.

La producción de leche diaria del total de vacas (en ordeño y secas), la producción anual y por hectárea aparecen en la Tabla 3. 
Se obtuvieron diferencias significativas $(P<0,001)$, ya que la producción de leche individual y por hectárea en las fincas con silvopastoreo casi duplicaron a las obtenidas en el testigo con monocultivo de gramíneas en condiciones de secano; a estos resultados contribuyeron el aporte de proteína y otros nutrientes de la leucaena en la alimentación de los animales, principalmente en los meses de mayor sequía. Por otra parte, los resultados en la producción de leche fueron la consecuencia directa de los índices reproductivos alcanzados por estos rebaños, los que se analizan a continuación.

Tabla 2. Producción de leche $(\mathrm{kg})$ y duración por lactancia (días)

\begin{tabular}{lccc}
\hline Sistema & $\begin{array}{c}\text { Lactancia 305 } \\
\text { días }\end{array}$ & $\begin{array}{c}\text { Producción } \\
\text { total/lactancia }\end{array}$ & $\begin{array}{c}\text { Duración } \\
\text { lactancia }\end{array}$ \\
\hline Silvopastoreo & $2572^{\mathrm{a}}$ & $2776^{\mathrm{a}}$ & $340^{\mathrm{a}}$ \\
Monocultivo & $2142^{\mathrm{b}}$ & $2229^{\mathrm{b}}$ & $318^{\mathrm{b}}$ \\
ES \pm & $91,25^{\star * *}$ & $98,5^{\star * *}$ & $7,99^{* * *}$ \\
\hline
\end{tabular}

a, $b$ Valores con superíndices no comunes difieren a $P<0,05$ (Duncan, 1995)

*** $\mathrm{P}<0,001$

Tabla 3. Producción de leche diaria y anual por unidad y por hectárea $(\mathrm{kg})$

\begin{tabular}{lccc}
\hline Sistema & $\begin{array}{c}\text { Producción } \\
\text { diaria }\end{array}$ & $\begin{array}{c}\text { Producción } \\
\text { anual }\end{array}$ & Producción/ha/año \\
\hline Silvopastoreo & $6,2^{\mathrm{a}}$ & $2216^{\mathrm{a}}$ & 3147 \\
Monocultivo & $3,6^{\mathrm{b}}$ & $1147^{\mathrm{b}}$ & 1790 \\
ES \pm & $0,2026^{\star \star *}$ & $112.8^{\star \star \star}$ & - \\
\hline
\end{tabular}

$a, b$ Valores con superíndices no comunes difieren a $\mathrm{P}<0,05$ (Duncan, 1995)

*** $\mathrm{P}<0,001$

En la Tabla 4 aparece el intervalo interpartal (IPP), los servicios de inseminación por concepción (SIC), el índice de producción láctea (IPL), que es la producción de leche promedio diaria obtenida entre uno y otro parto, y la natalidad alcanzada.

Los resultados evidencian la superioridad del silvopastoreo en el comportamiento reproductivo del rebaño en las unidades con la tecnología durante más de 3 años de explotación, donde el porcentaje de vacas gestantes 
superó en más de $12 \%$ al testigo. Este comportamiento contribuyó al incremento del número de vacas en ordeño, que varió entre 66 y 72\%, con lo que se logró duplicar la producción de leche en aquellas unidades donde se implantó la tecnología.

Tabla 4. Indicadores reproductivos, índice de producción láctea y natalidad

\begin{tabular}{lcccc}
\hline Sistema & IPP (días) & SIC & IPL (kg/día) & Natalidad \\
\hline Silvopastoreo & $423^{\mathrm{a}}$ & $1,92^{\mathrm{a}}$ & $6,5^{\mathrm{a}}$ & $84^{\mathrm{a}}$ \\
Monocultivo & $498^{\mathrm{b}}$ & $2,73^{\mathrm{b}}$ & $4,4^{\mathrm{b}}$ & $79^{\mathrm{b}}$ \\
ES \pm & $15,7^{\star \star \star}$ & $1,51^{\star \star \star}$ & $0,24^{\star \star *}$ & $0,025^{\star \star *}$ \\
\hline
\end{tabular}

a, b Valores con superíndices no comunes difieren a $P<0,05$ (Duncan, 1995)

*** $\mathrm{P}<0,001$

Por su parte, la composición bromatológica de la leche (\%) y el costo de producción del kilogramo de leche (pesos) se presentan en la Tabla 5. Las mayores diferencias significativas $(P<0,001)$ se alcanzaron en el porcentaje de grasa en la leche y los tratamientos con silvopastoreo fueron los más favorecidos. La misma tendencia se manifestó para los sólidos no grasos (SNG) y sólidos totales (ST); asimismo, la leche se cotizó a un precio medio de 0,47 pesos el kilogramo, con un máximo de 0,56 pesos en lo referente al precio de venta, lo que reafirma su buena calidad.

Tabla 5. Composición de la leche (\%) y costo por kilogramo (pesos)

\begin{tabular}{lcccc}
\hline Sistema & Grasa & SNG & ST & Costo \\
\hline Silvopastoreo & $4,32^{\mathrm{a}}$ & $8,45^{\mathrm{a}}$ & $12,67^{\mathrm{a}}$ & 0,43 \\
Monocultivo & $4,03^{\mathrm{b}}$ & $8,30^{\mathrm{b}}$ & $12,53^{\mathrm{b}}$ & 0,53 \\
ES \pm & $0,028^{* * *}$ & $0,019^{\star}$ & $0,018^{* *}$ & - \\
\hline
\end{tabular}

a, b Valores con superíndices no comunes difieren a $P<0,05$ (Duncan, 1995)

${ }^{*} \mathrm{P}<0,05 \quad$ ** $\mathrm{P}<0,01 \quad{ }^{* *} \mathrm{P}<0,001$

\section{EFECTOS ECONÓMICOS}

La evaluación de la eficiencia bioeconómica de cualquier sistema de producción animal debe contemplar el análisis de ciertos indicadores técnicos y de la 
situación financiera, entre los que se destacan las productividades por animal, por unidad de superficie y por niveles de insumos aplicados, y el comportamiento reproductivo de los animales, etc., los cuales son decisivos en el análisis de la sostenibilidad de la finca o unidad pecuaria (Reinoso, 2000).

Por ello, se presenta un balance financiero comparativo entre la tecnología del silvopastoreo y el monocultivo de gramíneas (Tabla 6).

Tabla 6. Algunos indicadores de eficiencia económica en ambos sistemas (pesos)

\begin{tabular}{lcc}
\hline \multicolumn{1}{c}{ Indicadores } & Silvopastoreo & Monocultivo \\
\hline Ingresos totales/ha & 3132,49 & 1287,47 \\
Ganancia (pérdida/ha) & 1332,49 & $(65,99)$ \\
Ganancia (pérdida/UGM) & 797,85 & $(53,65)$ \\
Costo/kg de leche producida & 0,51 & 0,81 \\
Costo/peso producido & 0,57 & 1,05 \\
Costos totales/ha & 1788,04 & 1353,47 \\
Costos totales/UGM & 910,29 & 1100,44 \\
Gastos de alimentos/costo total (\%) & 3,55 & 19,83 \\
Gastos de salario/costo total (\%) & 45,83 & 21,54 \\
Relación beneficio/costo & 1,59 & 0,95 \\
Umbral de rentabilidad & 73679,00 & 103191,60 \\
\hline
\end{tabular}

La tecnología del silvopastoreo alcanzó una relación beneficio/costo de 1,59 pesos, resultado que estuvo íntimamente asociado con la obtención de 0,51 pesos, lo que es inferior en 0,30 pesos por kilogramo de leche y en 0,48 pesos al costo por peso producido, al compararlo con el monocultivo. Esto significó producciones de leche superiores al umbral de la rentabilidad, la cual permitió cubrir con creces los costos totales de producción y dejar ganancias por hectárea y UGM, incluyendo los ingresos por nacimientos y ventas de animales sacrificados; mientras que los gastos en divisas en relación con el peso (moneda nacional) se exponen en la Tabla 7. 
Un desempeño favorable para el silvopastoreo presentó los indicadores de eficiencia económica expresados en USD; los costos por kilogramo de leche producida fueron los más bajos ( 0.11 pesos); mientras que para producir un peso (MN) solo fue necesario invertir 0,13 USD.

Tabla 7. Algunos indicadores de eficiencia económica expresados en USD

\begin{tabular}{lcc}
\hline \multicolumn{1}{c}{ Indicadores } & Silvopastoreo & Monocultivo \\
\hline Costo de leche producida & 0,11 & 0,29 \\
Costo/peso producido & 0,13 & 0,38 \\
Costos totales/ha & 399,97 & 487,30 \\
Costos totales/UGM & 203,62 & 396,20 \\
Gastos de alimentación/costo total (\%) & 9,53 & 41,30 \\
\hline
\end{tabular}

Estos resultados están avalados por el balance nutricional de los rebaños, que muestra con claridad cómo los sistemas arborizados cuentan durante todo el año con una fuente forrajera adicional, el follaje de L. leucocephala, cuyo contenido proteico varía entre 27 y $29 \%$ para la época de seca y 26 y $27 \%$ para la lluviosa, a lo cual se le unen otros atributos muy importantes de esta especie.

La disponibilidad de alimentos por época del año, proveniente de las fuentes endógenas de cada sistema, y aparecen en la Tabla 8.

Tabla 8. Disponibilidad de biomasa seca comestible (BMSC), PB y EM por época y sistema, proveniente de las fuentes forrajeras endógenas

\begin{tabular}{ccccc}
\hline \multirow{2}{*}{ Sistema } & \multirow{2}{*}{ Época } & \multicolumn{3}{c}{ Disponibilidad/animal/día } \\
\cline { 3 - 5 } & & BMSC (kg) & PB (g) & Mcal/EM \\
\hline \multirow{2}{*}{ Silvopastoreo } & Seca & 13,07 & 1266 & 27,33 \\
& Lluvia & 14,22 & 1639 & 28,21 \\
\multirow{2}{*}{ Monocultivo } & Seca & 11,27 & 959 & 22,55 \\
& Lluvia & 12,64 & 1023 & 25,01 \\
\hline
\end{tabular}

El efecto positivo de la asociación gramínea-leguminosa en el rendimiento de materia seca y la disponibilidad de biomasa seca comestible se corrobora con 
los resultados de la Tabla 8; se aprecia que en el sistema arborizado se encontraban las mayores disponibilidades de PB y EM por animal por día, lo que explica en cierta medida el mejor desempeño bioeconómico de los rebaños explotados en este sistema. Prueba de esto es la contribución a los requerimientos de los animales de las fuentes alimenticias endógenas, cuyos resultados se expresan en la Tabla 9.

Tabla 9. Contribución de las fuentes alimentarias endógenas al aporte total de PB y EM de la ración diaria

\begin{tabular}{lcccc}
\hline \multirow{2}{*}{ Sistema } & \multicolumn{2}{c}{ Estación seca } & \multicolumn{2}{c}{ Estación lluviosa } \\
\cline { 2 - 5 } & PB & EM & PB & EM \\
\hline Silvopastoreo & 97,03 & 90,43 & 97,63 & 91,02 \\
Monocultivo & 46,48 & 71,08 & 54,84 & 71,78 \\
\hline
\end{tabular}

Al analizar los resultados en la aplicación del balance nutricional, considerando los rendimientos lecheros individuales obtenidos en cada época del año, se puede constatar que el silvopastoreo muestra la menor dependencia de las fuentes exógenas para cubrir los requerimientos nutricionales de los animales, lo que significa que está en mejores condiciones de alcanzar su bioseguridad alimentaria, toda vez que se potencian las interacciones positivas del conjunto suelo-planta-animal.

Son múltiples los beneficios que el silvopastoreo puede aportar y entre ellos se debe destacar las posibilidades que brinda a la ganadería para propiciar el desarrollo de la reforestación. La siembra de árboles le permite al productor mantenerse del aporte económico diario del ganado y poder esperar la producción forestal; además, como producto de las podas se han obtenido producciones de 10 y $12,5 \mathrm{~m}^{3}$ de leña/ha de Leucaena y Albizia, respectivamente, además de postes, varas, madera y flores que contienen néctar para las abejas. 


\section{CASO 2. TRANSFERENCIA DE TECNOLOGÍAS EN LA PRODUCCIÓN DE SEMILLAS DE PASTOS EN CUBA}

Se considera que las semillas de algunos pastos naturalizados en Cuba, como el guinea ( $P$. maximum), fueron introducidas por los propios esclavos trasladados al país para fomentar los campos de caña de azúcar. Tanto es así que la historia recoge que las primeras cargas al machete durante las guerras independentistas se realizaron en campos de esa hierba, los cuales resultaban tan densos y altos que los mambises podían ocultarse a caballo y atacar a los soldados españoles. Esto demuestra la germinación y viabilidad de las semillas que diseminaron los animales y el viento. Sin embargo, en el desarrollo posterior de los pastizales, aún hasta el período 1970-1980, muchos ganaderos preferían establecerlos empleando sus partes vegetativas extraídas con azadas y picos en agotadoras faenas.

Las ventajas del empleo de la semilla botánica con respecto a la agrícola, demostradas hace algunas décadas atrás, propiciaron el desarrollo, el empleo y la generalización de las tecnologías de semillas en la ganadería. No obstante, este tema no ha escapado a las dificultades y exigencias de la transferencia de tecnologías.

\section{GENERACIÓN DE LAS TECNOLOGÍAS}

La EEPF "Indio Hatuey", desde 1975, intensificó la generación de tecnologías mediante la creación de un grupo de especialistas dedicados a ésta actividad; se lograron tecnologías completas para más de 15 especies y variedades comerciales de pastos, entre gramíneas y leguminosas rastreras, granos y árboles. Estas tecnologías abarcaron a: guinea, brachiaria, andropogon, rhodes, buffel, millo, stylosanthes, teramnus, glycine, dolichos, canavalia, leucaena, albizia y gliricidia, entre otras. 
Dichas tecnologías abordaron un conjunto de aspectos que corresponden al siguiente esquema:

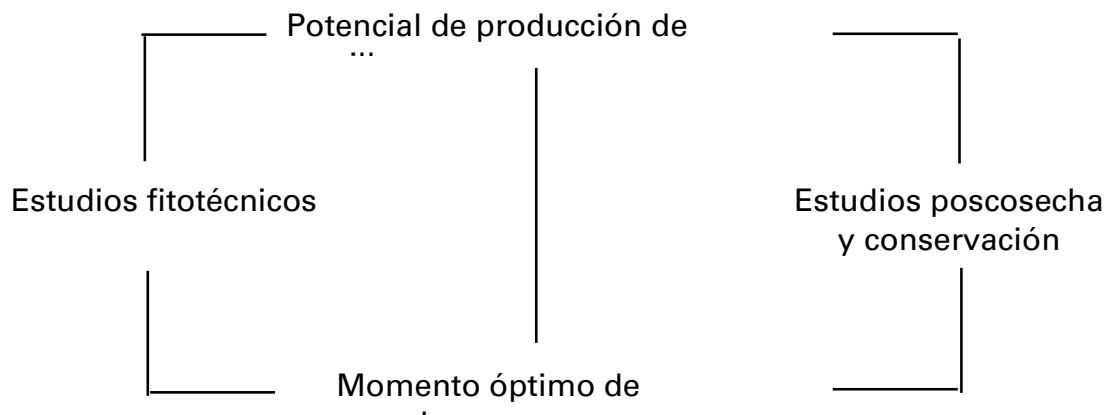

\section{Estudios realizados}

- Elección del lugar

- Momento de siembra

- Labores de limpieza y mantenimiento

- Fertilización orgánica

- Métodos de cosecha y manejo del campo de semilla

- Aplicación de fitorreguladores

- Combinación de producción de semillas con ganadería de leche y producción de carne

- Densidad, distancia y profundidad de siembra

- Fertilización NPK

- Control de plagas y enfermedades

- Latencia, dormancia y métodos de su interrupción

- Tipo de envases y almacenamiento

- Conservantes naturales

- Longevidad de las semillas 


\section{INICIO DE LA TRANSFERENCIA DE TECNOLOGÍAS}

La transferencia de tecnologías comenzó desde que se generaron las primeras conclusiones y recomendaciones de las investigaciones, en 1978, y no se esperó a que existiese una tecnología completa. A este proceso se le llamó, en aquel momento, extensión o introducción de resultados, donde se elaboraba un documento sobre el tema investigado, el cual se discutía en los consejos científicos y se presentaba a la Dirección de Ganadería; éste se aprobaba en una Comisión de Extensión, en la que participaban representantes de los centros de investigación, el Viceministerio de Ganadería y líderes de la producción pecuaria. Ejemplos ilustrativos fueron: "Densidad y método de siembra para la producción de semillas de guinea Likoni" y "Método de rejuvenecimiento para la producción de semillas de buffel".

En ambos casos se seleccionaron los lugares de aplicación donde las especies de gramíneas en cuestión tuvieron mayor aceptación; para la guinea Likoni ( $P$. maximum cv. Likoni) se seleccionó la provincia de Camagüey, donde estaba muy diseminada la guinea común, y para el buffel la Empresa "Iván Rodríguez", de la provincia de Guantánamo, por tener ésta provincia extensas áreas de dicha gramínea naturalizada en el lugar.

Para ejecutar esta labor de extensionismo se realizaron acciones vinculadas con la dirección de las empresas seleccionadas, y en ocasiones, se contó con la colaboración del personal técnico de las subestaciones del Instituto de Pastos y Forrajes del MINAGRI.

Estas primeras acciones colaboraron con la terminación y aprobación de las tecnologías comerciales, pero no significaron su completa adopción en cada uno de los lugares por diferentes motivos, como las largas distancias entre emisores y receptores sin suficientes medios de transportación; tampoco existía un verdadero receptor, ya que no había fincas semilleras ni las condiciones para 
adoptar las tecnologías, aunque pueden enumerarse otros factores que se señalarán en otro epígrafe. Las tecnologías fueron completándose para cada una de las especies y se desarrolló una conciencia en el sector ganadero acerca de la necesidad de la producción, beneficio y conservación de las semillas, por considerarse un factor limitante en el desarrollo de la ganadería en Cuba y en el trópico.

\section{PROCESO PARA LA DIFUSIÓN Y ADOPCIÓN}

En el enfoque planteado por Suárez y Pérez, (1999) en la Figura 2, no puede ser violado ninguno de los elementos si se quiere que las tecnologías transferidas alcancen una adopción para que los resultados sean irreversibles y perduren en el tiempo. Existen casos de fincas que, después de estar bien establecidas, ser rentables y consideradas entre las líderes del país, sufrieron un proceso reversible, y aunque hoy se recuperan, están lejos de los resultados que alcanzaron. Esto quiere decir que la cadena del personal directivo, los investigadores, así como el personal técnico y los obreros vinculados a la actividad, deben capacitarse constantemente para que puedan no solo adoptar, sino también mejorar las tecnologías generadas.

\section{FORMACIÓN Y CAPACITACIÓN}

En este proceso desempeñaron también un papel importante el Instituto de Ciencia Animal, el Instituto de Pastos y Forrajes, con su red de subestaciones, y diferentes instancias del Viceministerio de la Ganadería y subdelegaciones provinciales, así como la Asociación Cubana de Producción Animal (ACPA).

En este tema de las semillas se han generado dos tesis doctorales (en el ICA en 1980 y en 1984 en la EEPF "Indio Hatuey"), así como dos maestrías en esta última institución, y una tercera en el Instituto de Pastos, lo que ha contribuido al desarrollo de esta especialidad en el país. 
La EEPF "Indio Hatuey", para lograr una difusión de las tecnologías generadas, ha organizado cursos de posgrado aproximadamente cada 2 años, y ha impartido entrenamientos y cursos de capacitación a los productores. Ha contribuido, además, en la capacitación en cursos organizados por ACPA y financiados por organizaciones foráneas; asimismo, desarrolla un Programa de Formación de Maestros en Ciencias, del cual han egresado cuatro especialistas y se mantienen otros tres que aún no han defendido sus tesis.

En los cursos de capacitación y entrenamiento han participado especialistas de las 14 provincias del país, y de aproximadamente, 20 fincas de producción semillera.

En la adopción de las tecnologías ha sido importante la existencia de un receptor concreto, el cual es la finca semillera. En aquellos lugares donde ésta fue creada con un mínimo de condiciones, con suficiente autonomía y recursos propios y no vinculados a otras actividades de la empresa, se han obtenido resultados visibles, ya que existía un cambio de mentalidad del personal directivo, tanto en las subdelegaciones provinciales de la ganadería como en las empresas; sin embargo, donde no se logró una correcta capacitación de las personas vinculadas a la actividad, los intentos fracasaron o no se obtuvieron los resultados en correspondencia con el esfuerzo y los recursos invertidos. Un ejemplo de ello es la finca "La Rioja", creada en 1987 y perteneciente a la Empresa Pecuaria "Martí", en Matanzas; en las tablas 10, 11 y 12 se aprecian sus resultados productivos y la diversificación.

\section{DIVULGACIÓN CIENTIIFICA Y TÉCNICA}

Los resultados científicos que han generado las tecnologías de semillas han sido publicados en más de 100 artículos científicos, fundamentalmente en la revista Pastos y Forrajes de la EEPF "Indio Hatuey", y en dos monografías. Un vídeo, editado en 1991, que recoge todo el proceso tecnológico de producción, 
beneficio, conservación y control de la calidad, ha sido empleado en los cursos y entrenamientos; se generó un disco compacto, situado en una página web, que abarca las publicaciones fundamentales, las tecnologías y un vídeo de éstas.

También fueron publicados dos artículos técnicos en la revista ACPA, en 1984 y 1985, respectivamente, que recogen los resultados fundamentales alcanzados hasta esa fecha con las gramíneas comerciales guinea Likoni, rhodes callide, buffel biloela y buffel formidable.

Los resultados de las tecnologías se aplican hoy en más de 20 fincas de semillas distribuidas en todo el país, incluida la provincia de Matanzas donde se ubican tres fincas que han sido catalogadas como líderes por el personal directivo del MINAGRI.

En la provincia de Matanzas se han alcanzado más de 70 toneladas anuales de semillas y en pleno Período Especial, durante 1998, se han producido más de 40 toneladas, a pesar del déficit de recursos e insumos. Los costos de producción son aproximadamente de 60 centavos por peso, muy inferiores al precio que se comercializa en el mercado internacional por concepto de divisas, por lo que la semilla es un factible rubro exportable. Durante 1998, por el proceso de innovación tecnológica y el desarrollo de las fincas, basadas en la sustentabilidad, además de los ingresos por concepto de la semilla de 300000 pesos, se han obtenido otros ingresos debido al autoconsumo y la comercialización de productos agrícolas como el plátano y la piña.

Estas fincas han logrado ingresos por la venta de semillas a instituciones extranjeras, por lo que poseen cuotas de más de $\$ 12$ 000,00 USD para la adquisición de insumos destinados al desarrollo de las propias fincas. El forraje de estas áreas se ha empleado en el pastoreo directo para la ceba de toros para suministro de carne al turismo y se ha suministrado a las diferentes categorías de bovinos en las empresas donde están enclavadas las fincas de semillas, que 
son las áreas con mayor rendimiento por unidad de superficie. Esta carne, de alta calidad, se produce a muy bajo costo sin el empleo de divisas, a la vez que se ahorra el combustible y la maquinaria en el corte de forraje, práctica que es necesaria para efectuar posteriores cosechas de semilla. El uso mínimo de la maquinaria también tiene como beneficio que impide el apelmazamiento y la compactación de los suelos; mientras que la presencia de los animales en el consumo directo para la ceba contribuye a mejorar la fertilidad del suelo y sus relaciones bióticas. Este resultado es un aporte significativo a la reducción de la contaminación ambiental, al incremento de la rentabilidad de las empresas, la disminución de los costos y los suministros de productos de calidad para el turismo a muy bajo costo, ya que a esas áreas no se les aplica fertilización mineral ni pesticidas y es mínimo el empleo de la maquinaria. Debe agregarse que el hecho de no emplear piensos ni productos químicos en la ceba de toros, hace de su carne un producto prácticamente orgánico.

A modo de resumen, los elementos considerados importantes para la transferencia de tecnologías de producción de semillas de pastos en Cuba son:

- Se ha llevado a cabo un programa de investigaciones, durante más de 25 años, y se han generado más de 100 publicaciones científicas, lo cual condujo a la elaboración de reseñas tecnológicas para más de 12 especies de gramíneas y leguminosas herbáceas y arbóreas de importancia para la ganadería vacuna cubana y tropical.

- La organización de cursos y entrenamientos para investigadores y productores.

- Los resultados obtenidos se han transferido, en una primera instancia, a lugares localizados en empresas pecuarias durante más de dos décadas, para después analizarlos en el Consejo Científico de la institución y en los grupos de trabajo del MINAGRI. 
Tabla 10. Producción de la Finca de Semillas "La Rioja”, Empresa Pecuaria Martí

\begin{tabular}{|c|c|c|c|c|c|c|c|}
\hline \multirow{2}{*}{ Especies y cultivares } & \multirow{2}{*}{$\begin{array}{l}\text { Área } \\
2000 \\
\text { (cab.) }\end{array}$} & \multicolumn{6}{|c|}{ Producción (t) } \\
\hline & & 1991 & 1992 & 1995 & 1997 & 1998 & 1999 \\
\hline \multicolumn{8}{|l|}{ Gramíneas } \\
\hline Guinea likoni & 6,2 & 9,2 & 3,7 & 5,0 & 8,6 & 4,5 & 0,85 \\
\hline Rhodes callide & $1,0^{\star}$ & 1,4 & - & - & - & - & - \\
\hline Andropogon 606 & 0,6 & - & 0,2 & - & 1,1 & 0,23 & 0,4 \\
\hline B. humidicola & 0,1 & - & - & 0,05 & 0,06 & 0,03 & 0,05 \\
\hline B. decumbens Basilisk & 2,30 & 0,54 & - & $-0,35$ & 1,01 & 0,80 & 0,7 \\
\hline \multicolumn{8}{|l|}{ Leguminosas } \\
\hline Glycine & 1,0 & 0,75 & 1,0 & 0,35 & 2,8 & - & 0,11 \\
\hline Stylosanthes & 0,6 & - & - & 0,32 & 0,48 & 0.15 & 0,45 \\
\hline Teramnus Semilla Clara & 0,3 & 0,85 & 0,28 & - & - & 0,04 & 0,04 \\
\hline Centrosema IH-129 & 0,1 & - & - & - & - & - & 113 \\
\hline $\begin{array}{l}\text { Leucaena leucocephala } \\
\text { Perú }\end{array}$ & 1,3 & 1,04 & - & 0,75 & 0,83 & 0,01 & 0,04 \\
\hline \multicolumn{8}{|l|}{ Granos } \\
\hline Canavalia & 0,1 & - & - & 0,3 & 0,7 & - & 0,3 \\
\hline Sorgo forrajero & 1,0 & 1,23 & - & - & - & 1,0 & 0,5 \\
\hline Girasol & - & 7,0 & - & - & - & - & - \\
\hline Soya & - & 2,23 & - & - & - & - & - \\
\hline Dolichos & - & 2,23 & 0,10 & - & - & - & - \\
\hline $\begin{array}{l}\text { Sub total } \\
\text { Otras áreas }\end{array}$ & 14,6 & & & & & & \\
\hline $\begin{array}{l}\text { King grass, morera, } \\
\text { plátano, autoconsumo y } \\
\text { naves de tapado }\end{array}$ & 3,8 & & & & & & \\
\hline Total & 18,4 & 26,4 & 5,28 & 7,12 & 15,5 & 6,7 & 3,55 \\
\hline \multicolumn{8}{|c|}{$\begin{array}{l}\text { *Rhodes: Se dejó de producir en } 1991 \\
\text { - Una caballería = } 13,42 \text { hectáreas } \\
\text { Análisis económico (1999): } \\
\text { Gastos totales por las semillas (pesos): } 30 \text { 500,00 } \\
\text { Costo/peso de producción mercantil: } 0,62 \\
\text { Ingresos por semillas (pesos): } 44 \text { 210,00 } \\
\text { Ganancia (año 1999): } 18 \text { 000,60 } \\
\text { Producción de semillas (kg): } 3550,00\end{array}$} \\
\hline
\end{tabular}


Tabla 11. Venta de otros productos en la Finca de Semillas "La Rioja"

\section{Balance de producción y venta de toros (1999)}

\begin{tabular}{|c|c|c|c|c|c|c|c|}
\hline $\begin{array}{l}\text { No. de } \\
\text { animales }\end{array}$ & $\begin{array}{c}\text { Peso } \\
\text { promedio } \\
\text { inicial } \\
(\mathbf{k g})\end{array}$ & $\begin{array}{c}\text { Peso } \\
\text { promedio } \\
\text { final (kg) }\end{array}$ & $\begin{array}{c}\text { Período } \\
\text { de ceba } \\
\text { (días) }\end{array}$ & $\begin{array}{c}\text { Ganancia } \\
\text { diaria } \\
\text { (g/día) }\end{array}$ & $\begin{array}{c}\text { Ingreso } \\
\text { por } \\
\text { venta } \\
\text { (miles } \\
\text { de } \\
\text { pesos) }\end{array}$ & $\begin{array}{c}\text { Ganancia } \\
\text { (miles de } \\
\text { pesos) }\end{array}$ & $\begin{array}{l}\text { Ingreso } \\
\text { en USD } \\
\text { (miles) }\end{array}$ \\
\hline $\begin{array}{c}119 \\
\text { toretes }\end{array}$ & 298,3 & 460 & 300 & 538 & 109,2 & 25,23 & 52,2 \\
\hline \multicolumn{8}{|c|}{$\begin{array}{c}\text { Alimentación: Pastoreo de gramíneas, stylosantes y leucaena del banco de } \\
\text { semillas }\end{array}$} \\
\hline \multicolumn{8}{|c|}{ Venta de conejos } \\
\hline \multicolumn{2}{|c|}{ Cantidad } & \multicolumn{2}{|c|}{$\begin{array}{l}\text { Precio de venta por } \\
\text { conejo (pesos) }\end{array}$} & \multicolumn{2}{|c|}{ Período (meses) } & \multicolumn{2}{|c|}{ Venta (pesos) } \\
\hline \multicolumn{2}{|c|}{141} & \multicolumn{2}{|c|}{20} & \multicolumn{2}{|c|}{12} & \multicolumn{2}{|c|}{2820} \\
\hline
\end{tabular}

Alimentación: Banco de semillas de glycine, morera y subproductos de cítricos.

Viandas y hortalizas (plátano, yuca, tomate, ají y otros)

\begin{tabular}{ccccc}
$\begin{array}{c}\text { Viandas y } \\
\text { hortalizas }\end{array}$ & Producción (t) & $\begin{array}{c}\text { Precio promedio/kg } \\
\text { (pesos) }\end{array}$ & $\begin{array}{c}\text { Valor (miles de } \\
\text { pesos) }\end{array}$ & $\begin{array}{c}\text { Venta } \\
\text { USD }\end{array}$ \\
\hline & 88,1 & 0,46 & 40,35 & 420 \\
\hline
\end{tabular}

- Se ha considerado la innovación tecnológica como un proceso concebido en la propia unidad productiva, como producto del cual se han obtenido diversas modificaciones (las llamadas innovaciones incrementales) en las tecnologías de producción, beneficio y conservación.

- Después de una primera validación en las unidades seleccionadas, las tecnologías se extienden al resto de las fincas semilleras.

- Todas las etapas, desde la concepción de las tecnologías hasta su extensión, desempeñan un papel importante, pues un resultado establecido que no se monitorea sistemáticamente corre el riesgo de disminuir su impacto. 
Tabla 12. Estructura de la Finca "La Rioja", de la Empresa Pecuaria "Marti"

\begin{tabular}{lclc}
\hline \multicolumn{1}{c}{ Plantilla } & Cantidad & Maquinaria e implementos & Cantidad \\
\hline J' de Finca & 1 & Sembradora Saxonia & 1 \\
J' de Brigada & 1 & Maquina E-202 & 1 \\
J' de Recursos Humanos & 1 & Combinada E-514 & 1 \\
J' de Almacén & 1 & Combinada E-281 & 1 \\
Custodios & 4 & Tractores & 3 \\
Operador de maquinaria & 2 & Carretas & 2 \\
Operador de combinada & 3 & Fumigadora & 1 \\
Obreros $\quad$ agrícolas & 13 & Gradas & 1 \\
(semillas) & 1 & Empacadora & 1 \\
Montero & 1 & Bueyes (yuntas o parejas) & 5 \\
Oficinista & 1 & Implementos para bueyes & \\
Economista & 1 & Multiarado de bueyes & 1 \\
Obrero nave de conejo & & Cultivador & 2 \\
& 5 & Grada & 1 \\
Casa de tapado & 35 & Carretón de bueyes & 1 \\
\multicolumn{1}{r}{ Total } & & Arado & 1 \\
\hline
\end{tabular}

- Una inversión en moneda convertible que se realiza en una finca determinada puede no producir el resultado deseado. ¿Cuándo? Cuando la capacitación del personal es insuficiente, no se dispone de las tecnologías adecuadas, no se programa la adquisición de insumos según el presupuesto y no se tienen las técnicas capaces de explotar los medios asignados. La provincia de Matanzas es un ejemplo de cómo se pueden lograr resultados con una inversión más moderada, pero con las anteriores condiciones garantizadas.

\section{CRITERIOS PARA APLICAR LOS RESULTADOS}

1. Contar con la autorización y apoyo de la dirección de las empresas y del MINAGRI en las provincias.

2. Coordinar con los productores los planes de producción. 
3. Dichos planes no deben ser impuestos, debe predominar el criterio técnico y estar en dependencia de los recursos disponibles.

4. Los planes deben estar en función de las necesidades de la empresa, la provincia y el país.

5. Garantizar el mercado de las semillas.

6. Incrementar las exportaciones.

7. Lograr resultados que mejoren el próximo plan.

8. Defender y respetar las decisiones colegiadas y los logros.

9. Atención técnica sistemática a las fincas.

10. A partir de las ganancias, invertir y estimular a los trabajadores.

\section{CONCLUSIONES}

Gestionar el proceso innovador y la transferencia de tecnologías es clave para cualquier organización, y más aún en un centro científico, por ser factores determinantes del logro de una ventaja competitiva sostenible en el tiempo. Por ello, en la EEPF "Indio Hatuey" se ha desarrollado durante varios años un enfoque innovador para la generación, transferencia y mejora de tecnologías y conocimientos, el cual tiende a centrarse en las demandas de los productores.

Para ejemplificar dicho enfoque se utilizan estudios de casos relativos a dos tecnologías: el Silvopastoreo y la vinculación con la producción, beneficio y conservación de semillas, en las cuales se comenta el proceso seguido para su transferencia y los resultados en condiciones de producción.

\section{REFERENCIAS BIBLIOGRÁFICAS}

1. Anon. 2009. Gestión de la Innovación Tecnológica: Estudio de Casos. http://www.ceei-valencia.com/index.php?op $=8 \& n=1186$ [Consulta 5 de octubre 2010] 
2. Aait-El-Hadj, S. 1990. Gestión de la tecnología. La empresa ante la mutación tecnológica. Gestión 2000, Barcelona. 232 p.

3. Arbonies, A.L. 1993. Nuevos enfoques en la innovación de productos para la empresa industrial. Díaz de Santos, Madrid. 123 p.

4. Benavides, C.A. 1998. Tecnología, innovación y empresa. Pirámide, Madrid. 364 p.

5. Cazull I, M. 2009. Modelo, método y procedimientos de gestión de la transferencia de tecnología. www.gestec.disaic.cu/PONENCIAS2009/gestec/ Cuba/P30.doc [Consulta 5 de octubre 2010]

6. Drucker, P. 1954. The practice of management. Harper \& Row, New York. 201 p.

7. Drucker, P. 1986. La innovación y el empresario innovador. Edhasa, Barcelona. 427 p.

8. FAO. 1990. El estado mundial de la agricultura y la alimentación. Roma, Italia. 225 $p$.

9. FAO. 1993. Agricultura hacia el año 2010. Informe del 27 Período de Sesiones. Roma, Italia. p. 4

10. Gee, S. 1981. Technology transfer, innovation and international competitiveness. Wiley \& Sons, New York. 189 p.

11. Levitt, T. 1980. El éxito comercial mediante la diferenciación. Harvard-Deusto Business Review. 3:31

12. Mansfield, E. 1968. The economics of technological change. Norton, New York. 204 p.

13. Mesa, B. 1998. Silvopastoreo II. ACPA. 1:38

14. Morcillo, P. 1989. La gestión de la I+D. Pirámide, Madrid. 187 p.

15. Pavón, J.; Goodman, R.A. 1976. Proyecto MODELTEC. La planificación del desarrollo tecnológico. El caso español. Centro para el Desarrollo Tecnológico Industrial. Madrid. $87 \mathrm{p}$.

16. Piatier, A. 1987. Les innovations transectorielles et la transformation des entreprises. Conferencia sobre les regions, la innovación y tecnología. ESADE, Barcelona. $18 \mathrm{p}$.

17. Reinoso, M. 2000. Contribución al conocimiento del potencial lechero y reproductivo de sistemas de Pastoreo Racional arborizados empleando vacas Siboney de Cuba. Tesis presentada en opción al grado de Dr. en Ciencias Veterinarias. Universidad Central de Las Villas. Santa Clara, Cuba. 99 p.

18. Simón, L. 1999. Comportamiento del piñón florido (Gliricidia sepium) comparado con el algarrobo de la India (Albizia procera) en dos sistemas silvopastoriles. Pastos y Forrajes. 22:365

19. Simón, L. 2000. Manual práctico sobre el silvopastoreo. CIC-DECAP. La Habana, Cuba. 21 p.

20. Simón, L.; Lamela, L.; Esperance, M.; Reyes, F. 1997. Silvopastoreo. I. Transferencia tecnológica. ACPA. 2:31

21. Suárez, J.; Pérez, A. 1999. La gestión de la transferencia de tecnologías en la ganadería. Conferencia impartida en la Reunión Nacional de la Sociedad Cubana de Pastos. EEPF "Indio Hatuey". Matanzas, Cuba

22. Suárez, J.; Simón, L. 1996. El papel de los árboles forrajeros en el contexto socioeconómico y ecológico de la ganadería cubana. Taller Internacional Extensión'96. ETIAH. Holguín, Cuba 